\title{
5 Promoting eHealth literacy
}

\section{Integration of a health monitoring application and the support of a Digital Nurse}

\author{
Anna Haupeltshofer, Pascal Meier
}

\begin{abstract}
In Germany, the majority of the population has restricted health literacy. In this context, access to the Internet and new media is becoming increasingly important for information procurement. However, digitization is a challenge for society and nursing care. Against this background, the project Village Community 2.0 (Dorfgemeinschaft 2.0) concentrates on rural areas and human-technology interaction. As a solution, we designed the application FeelFit to strengthen eHealth literacy. The application helps users to monitor their health-related data in everyday life. Additionally, we developed a Digital Nurse, an educational health management concept. The Digital Nurse is a contact point for residents on health and technical questions. As technical developments often only concentrate on a single field, an interdisciplinary approach is necessary for a demand-oriented use of assistive technologies. We combined both concepts to offer older people the possibility to consult a nurse with an affinity for technology, who supports in the personal configuration of the app as well as in the operation and evaluation of the collected data and is sensitized about legal and ethical aspects in dealing with technology. Nurses can take on an informative and advisory function and close the gap between human and technology with their holistic view. In the article, we illustrate the integration of the developed concepts by a fictitious case study. Finally, the results are discussed regarding current research findings.
\end{abstract}

\subsection{Introduction}

The demographic change in Germany is characterized by the constantly increasing life expectancy of people, which is likely to lead to rising demand for social and health services in the future. Simultaneously, the number of health professionals, especially in the care sector, is declining, which makes high-quality and comprehensive health care more difficult, especially in rural areas (SVR 2014). This problem is also extended by the digital divide which describes, among other things, the differences in access to the Internet, Internet skills and experiences with the use of information and communication technologies (ICTs) (i.a. van Deursen and van Dijk 2011). The digital divide is particularly apparent for the group of people with medical conditions (Zhang et al. 2011). Against this background, major research funding programs have been launched in Germany in recent years in which research and funding focus on innovation and technology. A technological healthcare system has become a 
solution strategy (German Federal Ministry of Education and Research 2015). More and more technical assistance systems are being developed specifically for nursing staff and outpatient care to support people in need of care and their relatives. In the field of technical assistance systems development, the main goals are to maintain health and support older people to live longer safer and independently at home. The project Village Community 2.0 (Dorfgemeinschaft 2.0) concentrates in this context on rural areas and human-technology interaction. The project comprises an overarching research network with four interdisciplinary research groups (Nursing Science, Ethics, Information Systems, and Logistics). In addition, there are nine other consortium partners and more than 40 associated partners from the fields of local self-government, residents and business management. The project aim is to support people across generations with the use of digital and assistive technologies, to improve social participation (implementation of the inclusion and sharing economy). It also aims to bridge the digital divide between the different population groups in terms of access to and use of ICTs. Due to the infrastructure, such as long distances between residential buildings and educational and health facilities in the region, an online platform will provide digital services to support daily and community life. By linking the virtual space (platform) with the real living space, we hope to promote the social participation of people in their immediate environment and to support local care structures. Our project pilots in the rural area of Bentheim/ south Emsland (Lower Saxony) addressing the entire community concentrating in particular on older people and people in need of care.

To enable the use of the platform in the context of health management, we have developed the educational management concept Digital Nurse. The Digital Nurse is a contact point for residents on health and technical questions. We also developed FeelFit, a mobile health application. The application helps users to monitor their health-related data in everyday life. Many people already do so not only because of their interest in sports but also for health reasons (Bitcom e. V. 2018). By monitoring vital parameters, users can collect health data to participate in their health management. Since older people and people with chronic illnesses have lower health literacy (Schaeffer et al. 2016), we want to support these people in measuring their vital parameters if necessary. In this article, we will present both concepts and their integration answering the following research question: How can the concept of a health monitoring application and a Digital Nurse be integrated to improve the eHealth literacy of older people (65 years and older)?

To answer the research question, we will first present the background to eHealth literacy and socio-technical systems. In the following, we will outline the multi-perspective research approach from the perspective of nursing science and information systems and the resulting concepts Digital Nurse and FeelFit. We then present the integrated concepts based on a fictitious case study. Finally, we will discuss our results and their effects and point out the limitations. 


\subsection{Background: eHealth literacy and socio-technical systems}

It is important to note that technology development should not focus only on monetary all-in-one solutions. Furthermore, in this context, $54.3 \%$ of the population in Germany has limitations in health literacy (Schaeffer et al. 2016). One of the most frequently cited definitions of health literacy (Sørensen et al. 2012) is the one by the World Health Organization (1998) which describes it as "the cognitive and social skills which determine the motivation and ability of individuals to gain access to, understand and use information in ways which promote and maintain good health" (World Health Organization 1998 p. 10). This implies that the majority of the population in Germany faces problems finding, understanding, evaluating and using health-relevant information. In particular, older people and people with a migrant background, chronic diseases or a low net income have low health literacy (Zok 2014; Schaeffer et al. 2016). The Shanghai Declaration on promoting health in the 2030 Agenda highlights that health literacy is a socially relevant issue and is part of the context of lifelong learning, which should begin in school curricula (World Health Organization 2016). Obtaining health-related information and being able to use it for oneself also means being able to participate in society and act independently - older people are often excluded.

As the use of electronic and mobile health applications has increased in recent decades, the term health literacy has also evolved. Today, there are many definitions of eHealth all about technology (Oh et al. 2005). For Norman and Skinner (2006), contextual factors also need to be considered, such as the medium (electronic resources) and skills that are involved in obtaining health-related information addressing or solving a health problem. This goes beyond a narrow concept of health education and personal behavioral communication to address the environmental, political, and social factors that determine health. They developed the Lily model, which deals specifically with eHealth literacy. The model includes six equal components of core competencies (health-, science-, computer-, information-, media- and traditional/numeracy literacy). If competencies from all areas are available, a good eHealth literacy of the person is to be expected. The researchers point out that improving literacy is a dynamic process. In addition, there is a need for coordinated education, especially for older people (Norman and Skinner 2006).

While each of the different components creates unique challenges, coping with these challenges requires a synergistic approach. Such an approach makes it possible to develop effective digital health education programs for patients and providers. Since an application for increasing eHealth literacy requires a complex interaction between machines such as mobile devices and portable sensors, the user and the environment, it can be considered a socio-technical system (Emery and Trist 1960). This is also the case for most of today's systems. Socio-technical systems consist of two interdependent subsystems, the social and the technical subsystem. In most cases, only the technical subsystem is focused on the development process of the sys- 
tem (Eason 2001). However, it is necessary to consider both subsystems to achieve good performance and high utility. In the field of health monitoring systems, there is a rapid increase of interest in technical systems with wearable devices that have flexible functions, e.g. for fitness and medical applications (Liang and Yuan 2016). But these systems usually do not offer the necessary interaction possibilities such as voice input and output as well as good usability for every user. As a result, users, especially those with physical limitations or little computer knowledge, cannot use the system. Moreover, there is no support within the social subsystem for people who do not know how to interact with the system or how to interpret the information provided by the system.

\subsection{Multi-perspective research approach}

Since technological development is often only carried out on one side, an interdisciplinary approach is necessary for the demand-oriented development of assistive technologies. Our work focuses on improving the eHealth literacy of people who want to stay informed about their health status or have health problems and who need support in measuring and documenting vital parameters.

Complementary to behavioral science, which explains how and why information systems work, the design science of information systems focuses on creating artifacts for a specific need (March and Smith 1995). For design science in information systems research, Hevner et al. (2004) have developed seven guidelines. By following the guidelines an artifact in the form of constructs, models, methods, and instantiations is developed in cycles of building and evaluating. These so-called design cycles are supplemented by relevance cycles for meeting the environment's needs and rigor cycles grounding the research in and adding to the knowledge base (Hevner 2007). Our research is applying the design science research methodology by Peffers et al. (2007), which is based on the guidelines (Peffers et al. 2007). First, in our investigation, we designed two artifacts independently of each other. On the part of the nursing sciences the education management concept Digital Nurse (3.1) and on the part of the information systems, a system for adaptable wearable health monitoring system called FeelFit (3.2) was developed. On both sides, we used use cases to document and implement artifact development. Use cases describe the interaction of an actor with a system to accomplish a specific task. We have adapted the structure of use cases by Jacobson (1993) to our needs so that they can efficiently support interdisciplinary research. Following the development of the concept and the application, we will integrate both with each other pictured by the use cases. 


\subsubsection{Perspective nursing science}

In today's digital world, online consulting and social media are becoming increasingly important for both society and care (Risling 2016). In the context of digitization and technological progress in healthcare, it is clear that the structures and tasks of nurses will change in various areas. Nurses will take on new roles in this context, including digital knowledge transfer through inclusion and education to promote patients' health literacy (Mather and Cummings 2017). Currently, there are a lot of research projects for the use of technology and socio-technical arrangements in care. The paradox is that there is a lack of nursing science systematization and positions in Europe for technology development and design. For example, the field of nursing informatics is still in its early stages in Germany, in contrast to the professionalization and specialization that has gone on for more than 20 years in the USA and Canada. Related disciplines and politicians agree that there is a high need for tech-education for the health care professions, especially in nursing. On the other hand, the integration of competencies in the field of nursing informatics into the curricula of nursing education has not been enshrined in law or given more consideration. For example, the German occupational law for higher nursing education only stipulates as objects "to be able to transfer new technologies into professional action" (Federal Ministry of Health 2017, § 37, Para. 3, 3 PflBRefG).

Although international literature already shows that nurses take on different roles in the context of technology use, such as mediator (to access, review and evaluate information), advocate (for clinical judgment, privacy, and security) and supporter (appropriate use of a variety of ICTs) for care recipients and their relatives. In particular, they have an ethical and legal perspective and responsibility of the originary IT competencies ${ }^{19}$. Thereby nurses behave like advocates of system users by including them and their perspective and by aiming to enable them to use technology for their benefit (Canadian Association of Schools of Nursing 2015; Chung and Staggers 2014; Staggers, Gassert, and Curran 2001). In the German-language nursing science discourse, the use of technology in care settings is discussed against the background of a deprofessionalization or professionalization of nursing (i. a. Hielscher et al. 2015; Friesacher 2010). In the international debate, the authors Locsin and Purnell (2015) do not argue for or against increased use of technology in care but point out that carers can be a bridge to the technological world while maintaining the humanity of their patients. Among other things, it is discussed whether the health work of practitioners can and might be replaced by technology in the future.

19 The use of technologies is omnipresent in nursing practice, e. g. in e-documentation, monitoring patients, when reading laboratory parameters, when importing patient data, this can be understood as originary competencies. 
One thing is certain: nurses are often being torn between the medical dichotomy and mechanistic conception of humanity and their holistic nursing attitude and perspective of unity.

\section{Methods}

At the Osnabrück University of Applied Sciences, we have therefore opted for formative evaluation for the development of an education management concept to promote eHealth literacy. Methodically, we apply qualitative research methods, discussion forums and interviews combined with a systematic literature analysis.

We started with an analysis of the actual situation and needs. We conducted three focus group discussions with citizens $(n=58)$ and one with experts $(n=19)$, stakeholders from the regional healthcare and social system (persons of the district Emsland with the activity field care, ambulant care services, a health insurance company, nursing and social managements, senior citizens counseling and a nursing support point) to identify the needs related to health, technology, and education (Haupeltshofer et al. 2019). Additionally, we collected data by an international scoping review to identify the nature and scope in terms of technology and teaching roles and to map relevant literature in the field of nursing informatics competencies. We examined the research question: "What characterizes nursing informatics as an expanding field about the roles and competencies of nurses in technical appropriation processes of older adults to promote their eHealth literacy?" (currently under review). Based on the clustered competence profiles it is planned to design the training of the Digital Nurse in the future. In November 2019 we will start with narrative interviews with older people (65 years and older) about their tech-stories, to capture both experiences and self-perception with technology (use) and to gain new perspectives.

\section{Concept: Digital Nurse}

We have developed the Digital Nurse focusing on prevention, education, and technology. The concept is formative evaluated, which means that the research results are integrated into the conception over the entire duration of the project and influence the entire construct. The results of the analysis of the real situation and needs show that the citizens experience the construct of a good neighborhood as a care-network, but they perceive it as dwindling in the region. Also, there are age-related stereotypes and a negative perception of age. There are two fundamental fears: on the one hand, the fear of being alone and lonely in old age and of being dependent on other people. As a result, people suppress care topics and the need for care, which leads to a late confrontation with personal health management. On the other hand, the citizens were open to new forms of care in the discussion forums. They supported the concepts of a community nurse as the first point of contact and the preventive home visits. About assistive technologies, there is a high demand for information about an application, handling, and their benefits. Furthermore, there is a fear of anonymization through 
the use of technology, as citizens have repeatedly stated that relationships between people should not be reduced or replaced by technology (Haupeltshofer et al. 2019). For our concept development, it became clear that older people need a slow introduction and sensitization to technology. Only in this way can fears be reduced and personal benefit put in the foreground. In addition, we concluded that only by looking at the experiences and ideas of older people about technology we will be able to educate technology according to their needs. Furthermore, the literature research showed that nurses play different roles in processes of technical appropriation and already have competencies that need to be examined in the use of assistive technology.

The Digital Nurse concept includes that nurses introduce the users individually to the need-oriented technology and support them in dealing with technology. We planned to set up an institutional education and information center with a fixed place (if possible, this will be extended by a mobile unit as a visiting low-threshold offer), which will be supplemented by offers from virtual and non-virtual learning settings and methods. The Digital Nurse is a low-threshold point of contact to reduce inhibitions, for example, we plan the institution to be situated in a village center. Thus, it is possible to consult a qualified academic nurse directly, who will carry out health and tech-handling related individual anamnesis. In addition to theme-related basic and advanced training courses on how to use a tablet or workshops on how to use the digital community platform, the focus is on E-Learning and the use of eHealth applications. The basic principle is that those information units are communicated clearly and demand-oriented to older people. In addition, (tele-) consultation hours and webinars will also be offered. This will be supported by online visualization of existing local educational offers (like the social map / Sozialmap.com 2019), existing care and health services, but also, for example, the participation of the offers of selfhelp groups, which are digitally mapped and communicated by the Digital Nurse if necessary. Therefore, the concept aims to ease residents' access to health-related information and to promote their eHealth literacy.

\subsubsection{Perspective information systems}

The degree of digitization of the German population shows that the older generations are on the advance but still stand behind the younger generations. There are several influencing factors for it: younger people or people with a high level of education show a higher degree of digitization than less educated or older people (Initiative D21 e. V. 2019). In this context, it is clear to us that the further development of existing technologies should be based on the needs of users and nurses. It is important to consider the needs of the target group so that the application is used more regularly and successfully. Therefore, when developing the application, we used a process that is oriented to the user's requirements. We emphasize that the application does not necessarily have to be used regularly, but regularity helps to achieve the best results. 


\section{Methods}

We used the ISO standard 9241-210:2010 for the human-centered design of interactive systems to develop the FeelFit application (International Standardization Organization 2009). The first phase includes the planning of the human-centered design process by defining the topic and setting the general conditions for the design process. We formed a project team consisting of five graduate students and three $\mathrm{PhD}$ students for one year to develop the FeelFit application. The focus was on the development of an application that helps to measure vital parameters and to display and store them clearly and simply. In the next phase, we carried out a systematic literature review based on vom Brocke et al. (2009) to determine how older people deal with technology in general and with eHealth applications in particular (vom Brocke et al. 2009). The results of the literature were supplemented by a series of interviews with the target group on this topic (phase 2) to understand and specify the context. In order to structure the search and user requirements (phase 3), we created fictitious personas to describe different types of users in the target group. Besides information about the demographics and the bio of the user, the persona describes the goals, worries, and anxieties as well as the motivation and experiences with regard to the use of the application. Based on these results we produced the FeelFit application (phase 4). The application consists of various input and output devices which enable a multimodal interaction. In phase 5 we evaluated the application. Therefore, we set up a realistic setting. The participants $(n=54)$ (between 17 and 65 years old) used all functions and evaluated the application in a later questionnaire focusing on application experience (Meier et al. 2019).

\section{Concept: FeelFit}

FeelFit uses smartphones and wearable sensors to collect vital parameters. It processes and stores them in the user profile and enables users to retrieve the information not only via smartphones but also via other devices such as a smart speaker (voice only) and a smart mirror (visual only). The application includes a conversational agent, such as a chatbot or a virtual personal assistant, for example, Amazon's Alexa, which enables natural voice interaction. By providing a great variety of devices every user can decide which devices he or she wants to use. Further, the variety allows the user to interact with the system depending on the situation. FeelFit collects and evaluates health-related data (heart rate, temperature, blood pressure, among others) and informs users about their state of health. FeelFit can be used as a conversational agent which enables a more natural interaction than the current mobile eHealth applications (Seeger et al. 2018). This possibility supports people in the system. The user can configure the app to his or her needs and adapt it to possible diseases. Further, the user can link the profile to the personal healthcare provider to grant access who can then get access to the information via a web portal. 
The evaluation showed that the application improves the provision of health information to users in their everyday lives. Nevertheless, 57.4\% had never used digital technologies to measure vital parameters. The participants highlighted the multimodal interaction with the conversational agent and the mirror. However, the evaluation shows that users need to be trained in the use of new technologies such as the conversational agent. In addition to dealing with the technology, however, users must be trained to deal with the vital parameters, which are sensitive information, particularly to older people. This was especially clear in the case of a participant who had his pulse displayed in the mirror and remembered his stay in hospital. Therefore, we link the application with the concept of the Digital Nurse to use the application as a help system for supplying information and to integrate it into a socio-technical system.

\subsection{Integration of the concepts Digital Nurse and FeelFit}

After completing the first iteration of the design cycle by evaluating FeelFit in a real environment, we took stock. On the one hand, we had the challenge that especially older people had problems with the handling of the devices and a beneficial configuration of the application. In addition to this finding, we also found that it is necessary and useful for users to be provided with more information about the personal benefit and context significance in addition to the measurement data. This would have been technically possible by adding more health information and interpretation possibilities, but this would also lead to a very complex system. At this point, it became clear that system use to promote eHealth literacy is not possible in this way. There is a need for training in technical use, as well as support in the transfer of the collected data into daily life. This means that there is a need for a contact person who is an expert in health:

To illustrate the impact for citizens in the pilot region the following fictitious case study shows how users can benefit from the use of the mobile health monitoring application with the care and support of a Digital Nurse: 


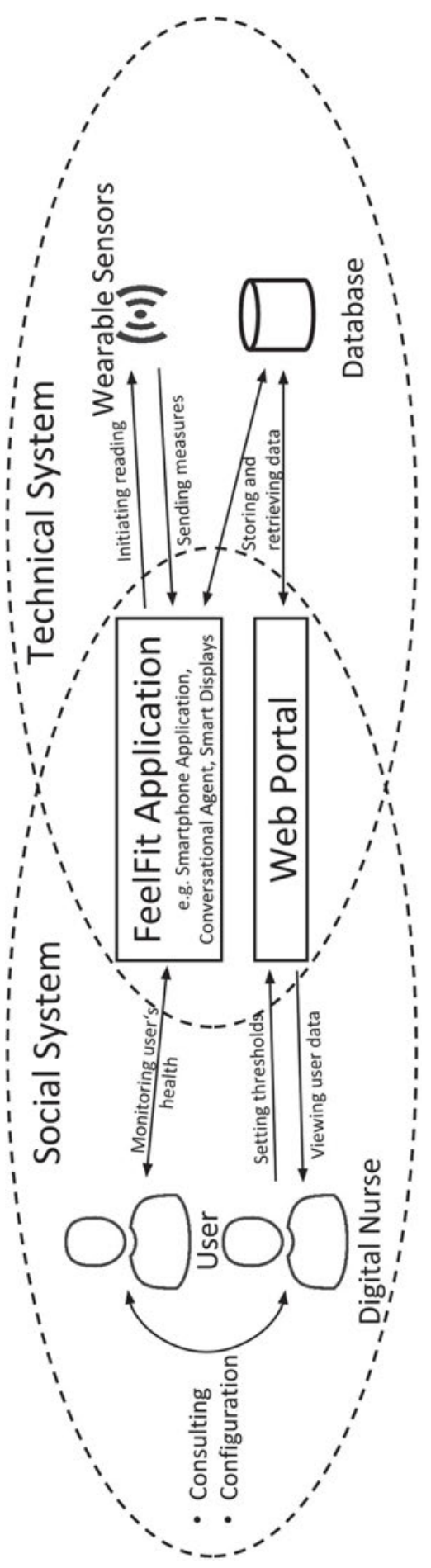

Fig. 5.1: Interaction of the socio-technical system. 


\section{Integrated Case Study}

Caroline Peters is 67 years old and lives alone in a house in a small village. She's had high blood pressure for five years. She treats her disease with antihypertensive drugs. In addition, the physician orders her to measure her blood pressure regularly to decide the proper medication. On the recommendation of her children, she buys a smartphone with a digital blood pressure monitor. Together with her daughter, she carried out the first installation, after which Mrs. Peters regularly used the smartphone to measure her blood pressure and store the data. However, there were changes in the application and an error message appeared. Mrs. Peters was confused and became insecure in the use of the smartphone. She was afraid of doing something wrong or unintentionally sharing her data with others. She asked her children to help her, but they were too busy. Mrs. Peters remembered the health kiosk near the town hall, where anyone can try out new technology and get support. The next day she spontaneously visited the kiosk and spoke to the Digital Nurse. After a tech-health anamnesis, the Digital Nurse recommends Mrs. Peters to buy a heart rate monitor and install the FeelFit application. The personal benefit for Mrs. Peters is that with FeelFit, the two sensors can be measured and clearly presented in a single application. They talk in detail about where personal data will be stored and which data protection settings are recommended. The Digital Nurse supports Ms. Peters in setting up FeelFit and adjusts the individual blood pressure and heart rate thresholds. She reminds Mrs. Peters that the application will tell her about exceeding the thresholds and will alert her if necessary. In addition, the Digital Nurse explains the possibility of voice commands in the FeelFit explanation. Afterward Mrs. Peters uses FeelFit for several weeks without complications. However, since the values more often exceed the threshold values, Mrs. Peters visits the Digital Nurse again who reports to Mrs. Peters and explains again that there are always normal deviations during measurements. She speaks clearly to her and agrees on a specific measurement value at which it is necessary to consult a physician. With the support of the Digital Nurse, Mrs. Peters feels safe using the technology and better informed about her health status.

As the use case example shows, the Digital Nurse's support intends to help people in the three areas of health, science and computer competencies or to compensate for a lack of skills in these areas.

\subsection{Discussion}

In rural areas with long distances to health and education institutions, the use of ICTs often means social inclusion and participation. Only those who can use health-related information in everyday lives can act independently and make health-related decisions; more than $10 \%$ of the German population have already experienced a pedometer and apps for measuring heart rate, blood pressure, etc. Besides, a further $27 \%$ are open for any use of these devices (Initiative D21 e. V. 2019). Even though more older people in Germany are online and the total number of offline people is falling from $25 \%$ to $21 \%$ compared to 2017 , the technical and digital competencies in computers of the over 70-year-olds are at a very low level (Initiative D21 e. V. 2018). Despite these numbers, eHealth literacy is rarely approached as a problem of the healthcare 
system. Illustrated by the case study, we mapped different levels of activity and interrelated scenarios. On the one hand, it is clear that a digital transformation of nursing is taking place and that the nursing profession will continue to change. On the other hand, there is a lack of political integration in the health system's laws and structures. Promoting health literacy is a nursing imperative (Speros 2005; 2011). We want to note that the lack of health literacy is a major problem in Germany and that there is an urgent need for action. At the national level, this task has not yet been taken over by nursing. In the context of health literacy, however, informed decision-making is playing an increasingly important role (Kolpatzik et al. 2018). In contrast, technology and the healthcare market are developing rapidly. The conditions for a successful demand-driven increase in health literacy must be examined in detail. For example, the digital divide must be considered from a country- and culture-specific perspective, as social disadvantage and a lack of digital infrastructure vary (i. a. Borgida et al. 2002; Denizard-Thompson et al. 2011). The technology introduction requires not only planning and patient education but also the development of cultural sensitivity for the technological values and attitudes of patients (Denizard-Thompson et al. 2011). The Digital Nurse takes on an informative and advisory function and closes the gap between human and technology with their holistic view. The practice-oriented reflections of international aspects of the field of nursing informatics and the perspective of the role of nurses in this educational context are therefore new findings for Germany.

The integration of both concepts should be seen as a work in progress approach that can eliminate many existing problems associated with the insufficient and unreliable health information supply as well as technology seen as an end in itself. One of the benefits of support is trust in oneself and in the application. The Digital Nurse as contact person increases the eHealth literacy by the support in the interpretation and reflection of data, the advice on privacy and data protection, but especially in the handling of the personal use of technology. Additionally, the focus is on demand-oriented technology development, but also on the individual ideas of the successful use of technology. The results of this study expanded the research in the field of socio-technical systems, through the integrated development of technical and social subsystems. This is also important because, for example, for the widely used eHealth literacy assessment (eHEALS) there is a late study the reliability and validity of which was only recently proven for older people (Chung and Nahm 2015), while previous literature has generally considered only one side, by studying either the use of technology in a social system or to adapt the social system to carry out a technology. Through the integrated approach, the socio-technical system can be examined holistically and adapted to the requirements of both subsystems jointly. 


\subsection{Conclusion}

This article presents a case study illustrating how the concept of a health monitoring application and a digital nurse can be combined to improve the eHealth literacy of older people (65 years and older). In our article, we have described different levels and possible challenges in using assistive technologies related to eHealth literacy; we have also developed an interdisciplinary demand-driven solution strategy. Using the illustrated perspectives of both scientific disciplines, we were able to explain why our scenario focuses on the development of FeelFit as well as on the specialized and faceto-face support of a Digital Nurse.

It can be limited that both concepts are not yet implemented in practice. In the prototype phase, for example, we used test accounts with anonymous health data. As part of further development, data security and data protection must be examined more closely, as health data are particularly sensitive and worth protecting. Also, in Germany the education of nurses in technology-related competencies is still in its early stages, so the integrated solution could not be evaluated under real conditions. As FeelFit is still a prototype, it should be examined whether people would use such a multimodal system to monitor vital parameters. Nevertheless, it should also be noted that for older people in technology-occupied settings there is an absolute need for practical and proactive scientifically evaluated approaches. However, the exchange with the use cases and real case scenarios ensure that both the application and the concept are coordinated. Future studies must then examine the long-term use of portable health monitoring systems with the support of a Digital Nurse.

Against the background of the social explosiveness of the subject area "assistance systems in an aging society”, we advocate for including a nursing science perspective in technology development. There is also a need to rethink health policy. Apart from the fact that eHealth literacy in Germany must be considered as the next decade of healthcare, the implementation of ICTs and assistive technologies alone is not enough. In particular, structures must be created to educate the population and health professionals in digital competencies.

\subsection{Acknowledgments and funding information}

Funded by the German Federal Ministry of Education and Research (Grant Number: 16SV7453). 


\section{References}

Bitcom e. V. Bundesverband Informationswirtschaft, Telekommunikation und neue Medien. 2018. Zukunft der Consumer Technology - 2018. Marktentwicklung, Trends, Mediennutzung, Technologien, Geschäftsmodelle. www.bitkom.org/sites/default/files/file/import/180822-CT-Studie2018-online.pdf. Accessed 18 December 2019.

Borgida, E., Sullivan, JL., Oxendine, A., Jackson, M-S., Riedel, E., and Gangl, A. 2002. Civic Culture Meets the Digital Divide: The Role of Community Electronic Networks. Journal of Social Issues 58 (1): 125-141. doi: 10.1111/1540-4560.00252.

Canadian Association of Schools of Nursing (CASN). 2015. Nursing Informatics: Entry-to-Practice. Competencies for Registered Nurses. https://www.casn.ca/wp-content/uploads/2014/12/Nursing-Informatics-Entry-to-Practice-Competencies-for-RNs_updated-June-4-2015.pdf. Accessed 18 December 2019.

Chung, S-Y., and Staggers, N. 2014. Measuring nursing informatics competencies of practicing nurses in Korea: Nursing Informatics Competencies Questionnaire. Computers, informatics, nursing CIN 32 (12): 596-605. doi: 10.1097/CIN.0000000000000114.

Chung, S-Y., and Nahm, ES. 2015. Testing reliability and validity of the eHealth Literacy Scale (eHEALS) for older adults recruited online. Computers, informatics, nursing CIN 33 (4): 150-156. doi: $10.1097 / C I N .0000000000000146$.

Denizard-Thompson, NM., Feiereisel, KB., Stevens, SF., Miller, DP., and Wofford, JL. 2011. The digital divide at an urban community health center: implications for quality improvement and health care access. Journal of Community Health 36 (3): 456-460. doi: 10.1007/s10900-010-9327-5.

Eason, K. 2010. Changing perspectives on the organizational consequences of information technology. Behaviour \& Information Technology 20 (5): 323-328. doi: 10.1080/01449290110083585.

Emery, FE., and Trist, E. Socio-technical Systems. In Management sciences, models and techniques, ed. Churchman, CW, and Verhulst, M. 83-97. London: Pergamon Press.

Federal Ministry of Health. 2017. Pflegeberufereformgesetz - PflBRefG.

Friesacher, H. 2010. Nursing Care and Technology - A Critical Analysis. Pflege \& Gesellschaft 15 (4): 293-367.

German Federal Ministry of Education and Research. 2015. Well care for the future: Start new research projects to support older people in rural and urban areas / We improve life in old age. www.bmbf.de/de/gut-versorgt-in-die-zukunft-1910.html. Accessed 18 December 2019.

Haupeltshofer, A., Blotenberg, B., Seeling, S., and Stutz, D. 2019. Mensch, Land, Pflege - wie gestaltet sich die Zukunft? Pflegewissenschaft 21 (9/10): 420-434. doi: 10.3936/1697.

Hevner, AR. 2007. A Three Cycle View of Design A Three Cycle View of Design Science Research. Scandinavian Journal of Information Systems 19 (2): 87-92.

Hevner, AR., March, ST., Park, J., and Ram, S. 2004. Design Science in Information Systems Research. MIS Quarterly 28 (1): 75-105.

Hielscher, V., Kirchen-Peters, S., and Sowinski, C. 2015. Increasing use of technology in nursing practice? Scientific discourse and practical applications in long-term care. Pflege \& Gesellschaft 20 (1): 5-19.

Initiative D21 e. V. 2018. D21-Digital-Index 2017 / 2018, Jährliches Lagebild zur Digitalen Gesellschaft: Eine Studie der Initiative D21. https://initiatived21.de/app/uploads/2018/01/d21-digital-index_2017_2018.pdf. Accessed 18 December 2019.

Initiative D21 e. V. 2019. Digital-Index 2018 / 2019, Jährliches Lagebild zur Digitalen Gesellschaft: Eine Studie der Initiative D21. https://initiatived21.de/app/uploads/2019/01/d21_ index2018_2019.pdf. Accessed 18 December 2019.

International Standardization Organization (ISO). 2010. Ergonomics of human system interaction-Part 210: Human-centred design for interactive systems. Switzerland. 
Jacobson, I. 1993. Object-oriented software engineering. A use case driven approach. Reprinted. Workingham: Addison-Wesley.

Kolpatzik, K., Schaeffer, D., and Vogt, D. 2018. Förderung der Gesundheitskompetenz. Eine Aufgabe der Pflege. In Agenda Pflege 2021: Grundlagen für den fachpolitischen Diskurs, ed. Szepan, N-M., and Wagner, F. 73-91. Berlin: KomPart.

Liang, T., and Yuan, J. 2016. Wearable medical monitoring systems based on wireless networks: A Review. IEEE Sensors Journal 16 (23): 1-1. doi: 10.1109/JSEN.2016.2597312.

Locsin, RC., and Purnel, I M. 2015. Advancing the Theory of Technological Competency as Caring in Nursing: The Universal Technological Domain. International Journal for Human Caring 19 (2): 50-54.

March, ST., and Smith, GF. 1995. Design and natural science research on information technology. Decision Support Systems 15 (4): 251-266. doi: 10.1016/0167-9236(94)00041-2.

Mather, C., and Cummings, E. 2017. Modelling Digital Knowledge Transfer: Nurse Supervisors Transforming Learning at Point of Care to Advance Nursing Practice. Informatics 4 (2): 12. doi: 10.3390/informatics4020012.

Meier, P., Beinke, JH., Fitte, C., Behne, A., and Teuteberg, F. 2019. FeelFit - Design and Evaluation of a Conversational Agent to Enhance Health Awareness. In 40th ICIS 2019: Proceedings of the International Conference on Information Systems - Information Systems at the Heart of Innovation Ecosystems, Munich, Germany, December 15-18, 1-17.

Norman, CD., and Skinner HA. 2006. eHealth Literacy: Essential Skills for Consumer Health in a Networked World. Journal of Medical Internet Research 8 (2): e9. doi: 10.2196/jmir.8.2.e9.

Oh, H., Rizo, C., Enkin, M., and Jadad, A. 2005. What is eHealth (3): a systematicreview of published definitions. Journal of Medical Internet Research 7 (1): e1. doi: 10.2196/jmir.7.1.e1.

Peffers, K., Tuunanen, T., Rothenberger, M-A., and Chatterjee, S. 2014. A Design Science Research Methodology for Information Systems Research. Journal of Management Information Systems 24 (3): 45-77. doi: 10.2753/MIS0742-1222240302.

Risling, T. 2016. Social Media and Nursing Leadership. Canadian Journal of Nursing Leadership 28 (4): 48-57. doi:10.12927/cjnl.2016.24561.

Schaeffer, D., Vogt, D., Berens, EM., and Hurrelmann, K. 2016. Gesundheitskompetenz der Bevölkerung in Deutschland: Ergebnisbericht: Universität Bielefeld, Fakultät für Gesundheitswissenschaften. doi: 10.2391/0070-pub-29081112.

Seeger, A.-M., and Pfeiffer, J. Heinzl, A. 2018. Designing anthropomorphic conversational agents: Development and empirical evaluation of a design framework. In 39th ICIS 2018: Proceedings of the International Conference on Information Systems - Bridging the Internet of People, Data, and Things, San Francisco, CA, USA, December 13-16, 1-17.

Sozialmap.com. 2019. Grafschaft Bentheim - Regionenübersicht. https://www.sozialmap.com/region/Grafschaft\%20Bentheim. Accessed 18 December 2019.

Sørensen, K., van den Broucke, S., Fullam, J., Doyle, G., Pelikan, J., Slonska, Z., Brand, H., and (HLS-EU) Consortium Health Literacy Project European. 2012. Health literacy and public health: A systematic review and integration of definitions and models. BMC Public Health 12 (1): 80. doi: 10.1186/1471-2458-12-80.

Speros, C. 2005. Health literacy: concept analysis. Journal of advanced nursing 50 (6):633-640. doi: 10.1111/j.1365-2648.2005.03448.x.

Speros, C. 2011. Promoting Health Literacy: A Nursing Imperative. Nursing Clinics of North America 46 (3): 321-333. doi: 10.1016/j.cnur.2011.05.007.

Staggers, N., Gassert, CA., and Curran, C. 2001. Informatics competencies for nurses at four levels of practice. Journal of Nursing Education 40 (7): 303-316. 
SVR, Sachverständigenrat zur Begutachtung der Entwicklung im Gesundheitswesen, ed. 2014.

Bedarfsgerechte Versorgung - Perspektiven für ländliche Regionen und ausgewählte Leistungsbereiche: Gutachten 2014. Bonn, Berlin.

van Deursen, A., and van Dijk, J. 2011. Internet skills and the digital divide. New Media \& Society 13 (6): 893-911. doi: 10.1177/1461444810386774.

Vom Brocke, J., Simons, A., Niehaves, B., Reimer, K., Plattfaut, R., and Cleven, A. 2009. Reconstructing the giant: On the importance of rigour in documenting the literature search process. ECIS (9): 2206-2217.

Wang, J-Y., Bennett, K. and Probst, J. 2011. Subdividing the Digital Divide: Differences in Internet Access and Use among Rural Residents with Medical Limitations. Journal of Medical Internet Research 13 (1). doi: 10.2196/jmir.1534.

World Health Organization (ed.). 1998. Health Promotion Glossary. Geneva, Switzerland.

World Health Organization (ed.). 2016. Shanghai declaration on promoting health in the 2030 Agenda for Sustainable Development: 9th Global Conference on Health Promotion, 21-24 November. Shanghai.

Zok, Klaus. 2014. Unterschiede bei der Gesundheitskompetenz: Ergebnisse einer bundesweit Repräsentativ-Umfrage unter gesetzlich Versicherten. WIdOmonitor 11 (2): 1-12. 